\title{
Application profile for Earth Observation images
}

\author{
Jean-Christophe Desconnets ${ }^{1}$, Hatim Chahdi $^{1}$, and Isabelle Mougenot ${ }^{2}$ \\ 1 Espace Dev IRD site de Lavalette, Montpellier, France, \\ Jean-Christophe.Desconnets@ird.fr, Hatim.Chahdi@ird.fr \\ 2 Espace-Dev, Université de Montpellier 2, Bâtiment 21, CC083 \\ F-91405 Montpellier Cedex 5, France, \\ Isabelle.Mougenot@univ-montp2. fr
}

\begin{abstract}
Based on the concept of an application profile as proposed by the Dublin Core initiative, the work presented in this manuscript attempts to propose an application profile for the Earth Observation images. This approach aims to provide an open and extensible model facilitating the sharing and management of distributed images within decentralized architectures. It is intended to eventually cover the needs of discovery, localization, consulting, preservation and processing of data for decision support. We are using the Singapore framework recommendations to build the application profile. A particular focus on the formalization and representation of Description Set Profile (DSP) in RDF is proposed.
\end{abstract}

Keywords: metadata, metadata standards, data interoperability, Dublin Core application profile, Earth observation

\section{Introduction}

The satellite image has become an essential source of information to address and analyze environmental issues quickly, repeatedly and in a reliable way. Available technologies for Earth observation satellite, offer a wide range of tools to address the needs of scientists and territory managers more accuratly. To facilitate their sharing and access, many initiatives are emerging from the community of Earth observation (space agencies, industry) or the broader environmental community, whether they are national $\left(\right.$ THEIA $\left.^{3}\right)$, European(INSPIRE ${ }^{4}$, COPERNICUS $^{5}$ ) or global $\left(\mathrm{GEOSS}^{6}\right)$. In most cases, access to the images is made possible by the deployment of a spatial data infrastructure that provides access to distributed and heterogeneous data [2]. It provides access to images through web services discovery, viewing and downloading, as well as online processing. These facilities require the implementation of an interoperability framework. The latter relies primarily on the adoption of a specific metadata standard from the user community or its variation. It is the basis for the implementation of image access

\footnotetext{
3 THEIA : French Land Data Center

${ }^{4}$ INSPIRE : Infrastructure for Spatial Information in the European Community

${ }^{5}$ The European Earth Observation Program

${ }^{6}$ Global Earth Observation System of Systems
} 
services. However, these systems are weakly interconnected and do not provide an as comprehensive view of available images as end-users could expect. Indeed, the process that led to these achievements is domain-specific and produces heterogeneous metadata schemes. That renders the implementation of common tools for image discovery difficult.

In addition, various interoperability frameworks are defined in the field of Earth observation. They offer metadata schemes that take the common needs of multiple users from various communities into account. The resulting scheme usually corresponds to the core elements of a standard. It provides general information about the resource to meet the needs of discovery and location. Those involving spatial characteristics (projection, resolution), acquisition parameters or quality (lineage, precision) are often absent from the proposed metadata. It will be difficult to foresee the extension of the functionality delivered by such a system without challenging underlying models or interoperability. To manage large sets of heterogeneous and distributed resources, this analysis leads us to propose a new approach to support the interconnection of geospatial resources from various communities. Many studies [5] have been conducted for several years to make different metadata standards interoperable and allow the conversion of various metadata sets from one standard to another and to ensure an efficient federated management.

Based on the concept of application profile as proposed by the Dublin Core initiative [6,7], the work presented in this manuscript endeavor to propose an application profile for the Earth observation images. Focused communities are those of earth observation and environment. This approach aims to provide an open model, extensible and usable, facilitating the sharing and management of distributed images within decentralized architectures. It is intended to eventually cover the needs of discovery, localization, consulting, preservation and processing of data for decision support. As a first step, our approach proposes a state of the art, which introduces the concept of satellite image and the analysis of appropriate standards for the description of geospatial resources. We also provide a brief overview on the role of metadata in the sharing of resources in the area studied. The notion of application profile will also be introduced. The following section presents the application profile through the various stages of its building. We identify functional requirements, describe the domain model and illustrate by examples, the formal constraint model named DSP (Description Set Profile) associated with the definition of our application profile.

\section{State of the art}

\subsection{Earth observation image}

In the context of our work, the resources ${ }^{7}$ that we want to describe are images from the Earth observation. These resources are acquired by artificial earth satellites, equipped with various sensors. They ensure the acquisition of an image of

\footnotetext{
7 The term resource is taken in its broadest sense, as any concrete or abstract entity, which may be identified, named, manipulated across multiple representations
} 
a part of the Earth. This acquisition is performed according to an acquisition characteristic for each sensor, which that will depend on the nature of the acquired image, such as spatial resolution. It is noted that an image is a large digital resource. For example, a very high resolution ${ }^{8}$ image on an area of $20 \mathrm{~km}$ by $20 \mathrm{~km}$ has a volume of several gigabytes.

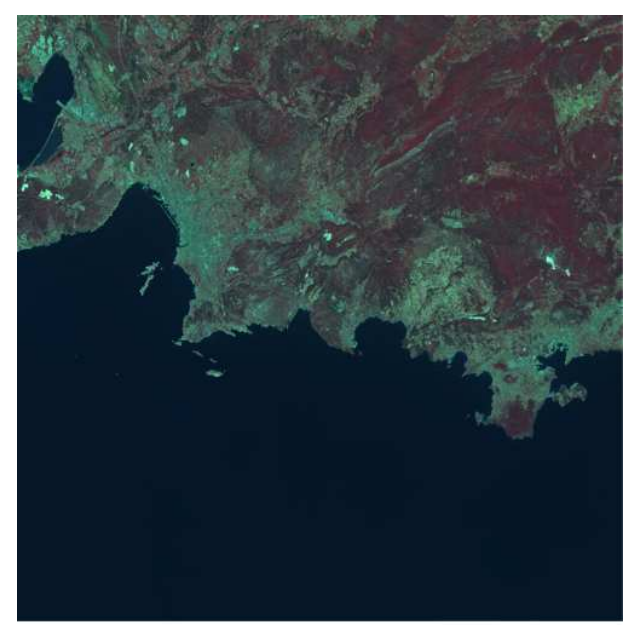

Fig. 1. SPOT 5 image representing a portion of the mediteranean sea (East zone of Marseille city), 10 meters of resolution. (source : équipex-Geosud project)

\subsection{Metadata standards for Earth observation}

Different standards, general or dedicated to a particular discipline, play essential roles facilitating the distributed resource management in decentralized architectures. After specifying the content and the added value of the standard Dublin Core, the section presents current standards dedicated to the description of both geospatial resources and Earth observations.

Dublin Core The Dublin Core standard has a general scope that goes well beyond the sharing of satellite images. Indeed, it has been proposed to provide evidence to generically describe any type of resource. Thus, it provides efficient ways to help discovery in the context of the web for any communities. For this purpose, Dublin Core has a fifteen elements description, which constitutes the core of standard. In our context, Dublin Core is used by the Discovery Service OGC CSW [19] to define the searchable elements (queryable elements) of the service in a generic way and could provide also a simplified view of retrieved results.

\footnotetext{
${ }^{8}$ the basic unit of the image represents a portion of space less than 1 meter on Earth
} 
ISO 19115 and ISO 19115-2 ISO 19115 standard [11], enacted by ISO $\mathrm{TC} / 211$ is the metadata standard for geospatial resources. It proposes a conceptual framework for describing these resources. It was designed to cover very large geographic community needs that extend from the data management to dissemination through their processing. Represented with the UML object formalism it includes twelve main packages, nine of which are common to all geospatial resources. Several packages are dedicated to the description of the spatial dimension of the resources, such as Extent Information, Spatial Representation or Reference System Information.

The ISO 19115 standard defines core elements. It corresponds to a minimum set of elements considered essential to meet the needs of discovery and location of a resource. Most of time, these core elements are used to provide metadata interoperability in information systems, which are pooling geospatial resources. The ISO 19115 standard has been extended by the ISO standard 19115-2 [13] to support the description of spatial gridded resources, such as satellite images. It provides new elements of description, such as the information on the platform and acquisition sensors. It defines some other to complete existing descriptions, especially to clarify the characteristics of processing performed on an image (Lineage Information package). Thus, it expands the scope of the ISO 19115 standard to meet the specific needs of image producers. The operationalization of the standards is guided by the ISO 19139 specification [9] which gives the transformation rules for serialization of metadata in XML format.

Earth Observation Metadata profile of Observations \& Measurements Focused on the specific needs of the Earth Observation community, the metadata profile Observation and Measurement $(\mathrm{O} \& \mathrm{M})$ [10] was built [14]. It is part of the HMA interoperability framework defined by the ESA ${ }^{9}$. It aims to facilitate the sharing of Earth observation products, whatever the mission and the sensor from which they come. In this context, the concept of metadata profile is the one proposed by ISO [12] and is quite different in these principles to the one presented in section3. It describes a metadata scheme and writing rules in XML, needed to describe the metadata of Earth observation products. More specifically, the metadata scheme is designed for the products from the general description to the more detailed observation mission (e.g SENTINEL) features provided with the acquisition of the image by one of these platforms. For this the model defines three levels of description: a "general" level on main characteristics of the products, a "thematic" one, which extends the previous one to describe the specific characteristic thematic products, such as optical, radar or atmospheric. The last one, the "mission" level, extending the previous one, describes the specific products of a mission, like those of the European mission SENTINEL ensuring the acquisition of radar images. Thus, this metadata scheme provides new metadata descriptions, related among others to acquisition parameters of an image, such as pitch, roll, yaw of the acquisition platform.

\footnotetext{
${ }^{9}$ ESA : European Spatial Agency
} 
They are essential to the geometric corrections made on the images after their acquisition.

\subsection{Role of metadata for sharing and access to Earth observation data}

Scientific, institutional and community initiatives for implementation of information systems to facilitate the discovery and access to the images are numerous. In Earth observation domain, the most emblematic is the system of the Group of Earth Observation Systems: GEOSS. On a global scale, GEOSS aims to provide decision support tools for a very wide range of users. The system of systems interconnects different observing systems, production or dissemination of data from satellite imagery.

The interconnection of different systems is based on a common set of standards including ISO 19115 [4]. It is used to provide a common metadata scheme for the description of resources aggregated by the systems of the system. The ISO 19115 scheme is used as a switch-across model for processing different metadata formats. It is also used to allow a uniform query of all descriptions via a discovery service OGC CSW [19]. It is based on the ISO19115 standard core elements. They are extended to describe the data access services, such as Web Map Service. If this model is relevant to the expected functional requirements, it offers a very limited capability to filter the large amount of data referenced by GEOSS. The heterogeneity of vocabularies from different descriptions is not considered and often leads to unsatisfactory accuracy of results. The THEIA Land Data Center provides geospatial resources from images at high and very high resolution for scientific community and public actors at the French national scale. It consists of a federation of data centers and processing units that diffuse their data via web services. The metadata have a similar role. At federation level, a specific abstract model inspired by the OGC specification called EO OpenSearch [20], is used to harmonize the harvested metadata records and represents them in a uniform manner to perform homogenous queries. Minimalistic, in order to simplify the operations of metadata harmonization and to meet the needs of image location, this abstract model focuses mainly on describing the context of data production and the main features of the image. Information about the data content and quality are not considered. A query on the content of the original image (spectral bands, spectral resolution) or on elements of his lineage can not be achieved.

European project GENESI-DR ${ }^{10}$ and GENESI-DEC ${ }^{11}$ could be considered relevant for our goals. Similar to the others, but with a clear focus on multidisciplinary issue, the objectives are also to facilitate access to Earth observation data. For this purpose the project has implemented a spatial data infrastructure

\footnotetext{
${ }^{10}$ GENESI-DR: Ground European Network for Earth Science interoperations - Digital Repositories

${ }^{11}$ GENESI-DEC: Ground European Network for Earth Science interoperations Digital Earth Community
} 
federating many heterogeneous databases. In addition, the infrastructure provides a set of customizable services that offers users the opportunity to compose their own processing [3]. Moreover, metadata play as the role of descriptors for the purposes of discovery and data processing services. In this multi-disciplinary context, the description of resources is not domain-specific but addressed by the use of the Dublin Core vocabulary. It extended to specific geospatial data descriptors, such as dclite $4 \mathrm{~g}$ : resolution, dclite $4 \mathrm{~g}$ : projection. Indeed, the metadata scheme DCLite4G [15] are built on the recommendations of the Dublin Core Metadata Initiative, namely on application profile principles [8]. Thus, the proposed model overcomes the community standards as they are from the geographical, the biodiversity and climate change community. The achievements of these projects address in part our goals. We want to extend the functional scope and take advantage of the use of the interoperability framework provided by RDF, including associated RDF vocabularies of geospatial domain.

\subsection{Notion of application profile}

General notions A potential reproach to metadata standards is that they have been designed independently of each other and thus are not able to meet all the information needs. For this purpose, application profiles reuse metadata standards to respond either to new requirements, or to more specific ones, for example to combine information from different sources to deliver new interpretations or to apply different filters on information. The principle is an open approach, by taking different elements of different metadata standards and combining them in a "mix and match" manner [6] to generate a new organization of metadata elements particularly suitable for the target application. The construction of an application profile is expected to meet the needs of discovery, characterization and consultation of distributed and heterogeneous resources, to cater to the application needs of a specific community.

Building principles The definition of an application profile is subject to different rules: a first principle is to rely on existing metadata standards, or to maintain an open and long term a new metadata standard, which covers the newly introduced metadata elements. The following principles relate to the publication of enrichment approaches and use of model entities from metadata elements. We use the work developed around the Singapore framework and application profile named DCAP (Dublin Core Application Profile) by the Dublin Core community. Methodological recommendations [18] and the specification of UML conceptual models facilitate construction activities of an application profile. A first structural model called DCAM (Dublin Core Abstract Model) [23], emphasizes the notion of resource. Its specialization on described resources is a collection of property-value pairs. The value is sometimes envisaged as a labeled resource and can be taken from controlled vocabularies.

A second structural model named DSP (Description Set Profile) [18] complements the DCAM model to provide a prescriptive framework for the construction 


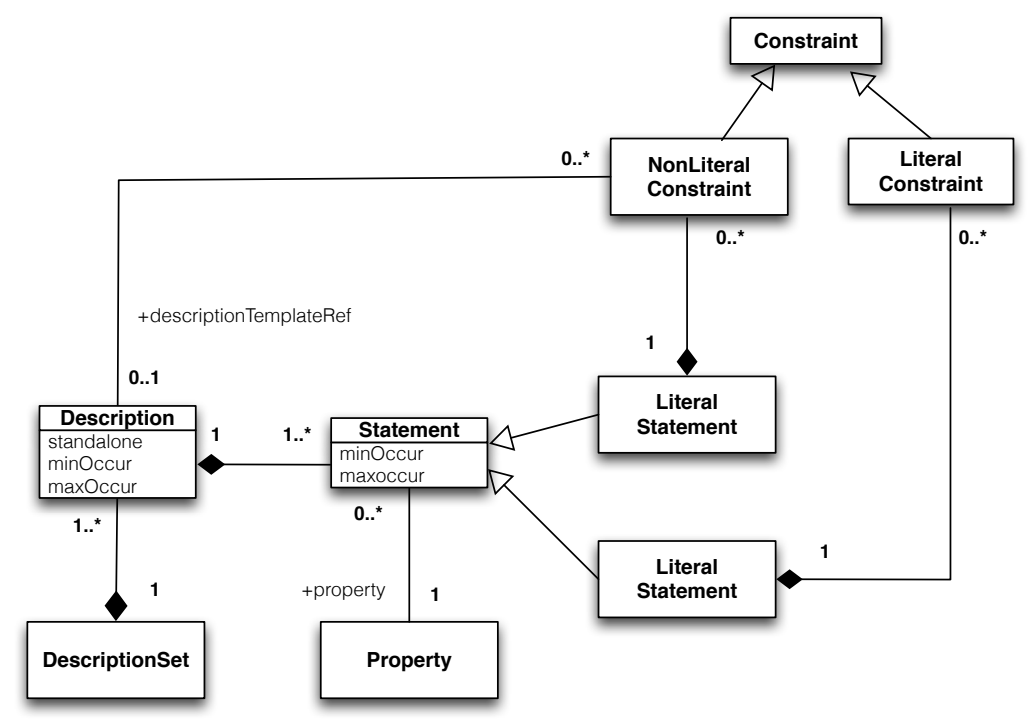

Fig. 2. Simplified UML Class Diagram of Description Set Profile

of the application profile. An application profile is then considered as a set of descriptions, and described through the concept of DescriptionSet Template. Each description is called DescriptionTemplate. It enriches a resource of interest in a decentralized manner and documents it through metadata elements from appropriate standards. These elements, as well as the different syntactic and/or semantics constraints that apply, are structured in statements called Statement Template. Statements are either Literalstatement Template, when metadata elements relate to literals or NonLiteralstatement Template when metadata elements relate to URI labeled resources. Constraints are explained through the concept of Constraint, which specialize in LiteralConstraint and NonLiteralConstraint.

Figure 2 shows a simplified structural diagram for the DSP, largely inspired by diagrams described in $[18,24]$.

\section{Earth Observation Application Profile}

\subsection{Background}

Our work takes place in the context of a project distribution of satellite images, the Equipex Geosud project [16]. Developed under the observation of use of spatial data by public French actors working on the management of natural areas and their resources, one of the objectives of this project is to set up a technical device to make high resolution satellite images accessible to users of heterogeneous skills, from non-specialist to remote sensing expert. These images come from 
different platforms and acquisition instruments. They are delivered in specific formats, whether the image itself, or the associated metadata. Furthermore, the spatial data infrastructure is part of the French national data center, the THEIA pole (cf. section 2.3), with which it has to interconnect. The figure 3 shows an

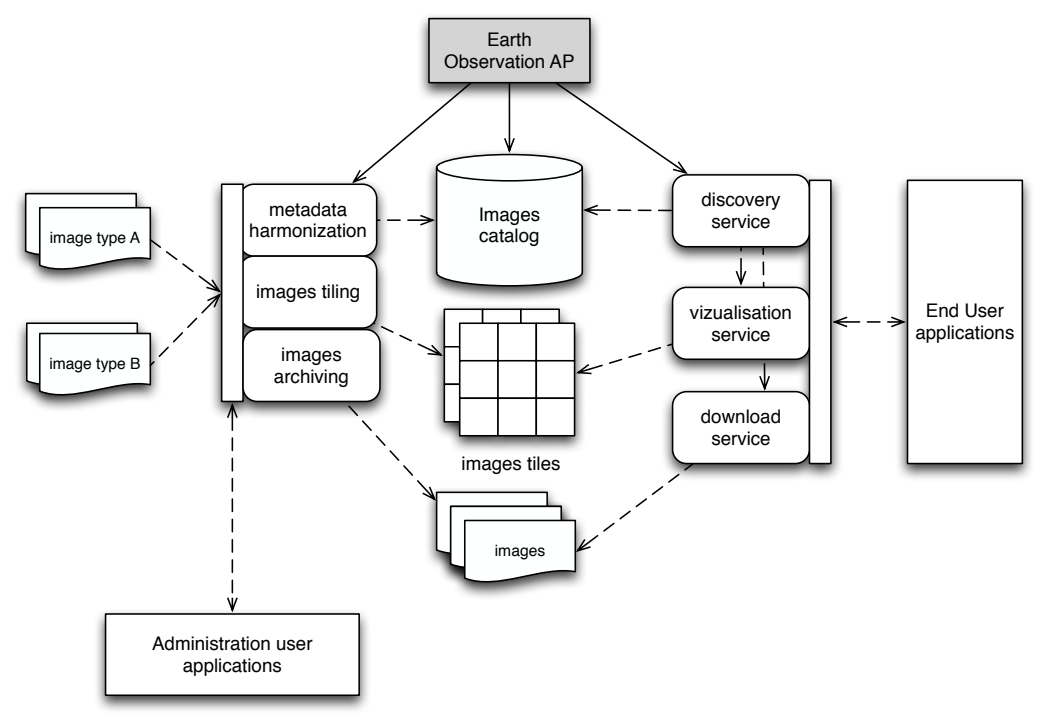

Fig. 3. Spatial data infrastructure architecture and application profile position

overview of the architecture of our system. It is based on components ensuring harmonization of image metadata, their tiles and their archives. The web user services : discovery, viewing and downloading are based on the image catalog. It provides the descriptions to search available images to invoke their visualization and consultation of their detailed characteristics. It also gives information to locate and download them. The application profile is a core element of our system. It provides descriptions which the metadata transformations rely on. It has a central role in the image catalog by structuring stored metadata. Finally, image access services build their queries on descriptions of images formalized through the application profile.

In a second step, it is planned to lean against user applications, designed to generate environmental indicator maps for the monitoring of natural resources. In this context, the application profile will also provide the descriptions required for running business processes.

\subsection{Approach}

The methodological approach proposed by Dublin Core metadata initiative finds its justification to overcome the heterogeneity of images from different providers. 
One of the main objectives is to offer uniform and semantically accessible image metadata to the target community. In addition, the ability to reuse different existing standards allows taking over the broad functional spectrum that we want to meet. Following the recommendations of the Dublin Core community, and particularly those encouraged by the Singapore framework, we present in this section the functional requirements on which our profile is based. Then, we describe the domain model related to context dissemination and processing of satellite images. Finally, we give some elements of the Description Set Profile (DSP) that ensures the implementation of the application profile in our infrastructure and a part of a DSP is proposed.

Identifying functional requirements According to the goals of our infrastructure and the user community, which it addressed, the main functional requirements are the following:

- Carry out a decentralized data description management to mobilize images only for download,

- Solve the heterogeneity of metadata schemes and terminologies to ensure a uniform query of an image catalog,

- Have a comprehensive model for describing images that can be used by the different access and processing services, but also for the administration of data (management life cycle, permanent archiving, monitoring data consumption by users, ...),

- Enrich vocabulary metadata to be accessible to non-specialist users, including adding new descriptions about the content of the image (e.g land cover range in the image),

- Offer a discovery service for images at different levels of granularity (collection, feature) to facilitate the discovery in a large amount of data,

- Search and processing of the images within the infrastructure,

- Provide indicators of image quality (e.g geometric accuracy) to allow the user to evaluate the image quality himself.

Domain model A domain model is a conceptual model that identifies the entities that we want to describe and the relationships between them, according to functional requirements. Its object representation using UML formalism allows to share our point of view with the different participants in your system, whether they are information system specialists or not. The proposed model generalizes and extends the existing models within the geographical community, including the one proposed by the Public Geospatial Data Project FGDC [21] and its specialization by GENESI-DEC (cf. section 2.3) to establish an application profile: DCLite4G [22].

Our model is shown in figure 4 . The core entity Resource refers to the concept of Resource as proposed by the DCAM (Dublin Core Abstract Model). This is an abstract entity that represents all the resources that are shared. It generalizes the two types of specific domain resources, namely the entities Process and EarthImage. The reflexive relationship isPartOf the Resource entity 


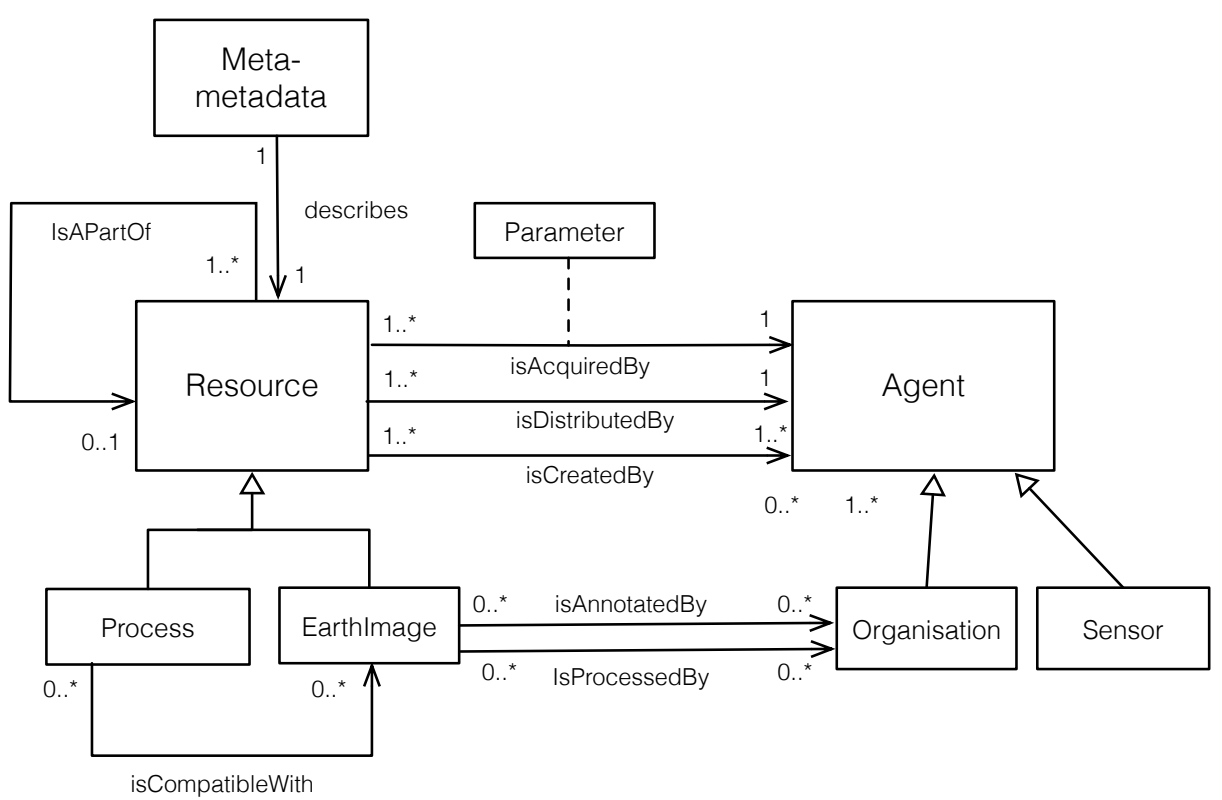

Fig. 4. Domain model for Earth Observation (UML Class diagram)

can represent, for EarthImage resource type, aggregation relationship between a collection of images, all images having common properties, and an image. Semantically equivalent to the Class dcmi: Agent, the Agent entity is an abstract entity, which is specialized in Organization and Sensor. The first describes the institutions involved in the creation of a resource or its distribution within the community through relationships isCreatedBy, isDistributedBy.

The second Sensor, describes the instruments used to acquire a satellite image. The isAcquiredBy association allows to describe the characteristics of the sensor used to acquire an image or set of images, as well as the parameters for the acquisition of an image (association class Parameter).

The entity EarthImage represents the Earth observation images that we want to make accessible or process. It provides the intrinsic characteristics of the image including information on the temporal and spatial extent, its spatial and spectral resolutions and information to ensure its distribution, such as size and conditions of use. Its characteristics are completed by semantic annotations relating, for example, administrative entities covered by the image or the land cover classes identified on its spatial extent. These annotations are intended to facilitate the discovery process by non-expert users.

The entity Process represents unit processing or processing chains applicable to Earth observation images. They correspond to basic operation support, for instance, to extract a portion of an image, to calculate a vegetation index or to more complex processing leading to the creation of a new resource, such as a 
map indicator of natural resources. This entity describes the input and output in terms of size or type of data, and the nature of the parameters required to run it. The relationship is CompatibleWith to associate an image or collection of images to processing and specifying the compatibility of a processing to a collection of images. Finally, Meta-metadata entity provides the information required for the management of metadata records (e.g modification date, description language, etc..).

Description Set Profile According to the proposal of Singapore Dublin Core framework and formalization of description constraints language proposed by $[17,18]$, each of the entities identified in the domain model are divided into sets of description. In a first stage, we have focused our efforts on describing a few features of interest to cover the needs of discovery and location. Description of the Template EarthImage and Organization classes are available at this address: http://purl.org/eoap/. For clarity they are serialized using the RDF N3 syntax [1]. The properties that describe our interest entities refine elements from basic description of DCMI Metadata Terms. They are refined to describe the characteristics of spatial data, such as spatial footprint of an image by the ISO 19115 metadata standard.

We provide an extract from Template Description EarthImage_T on the refinement of the element dcterms : coverage by iso19115 : EX_GeographicBoundingBox coverage class.

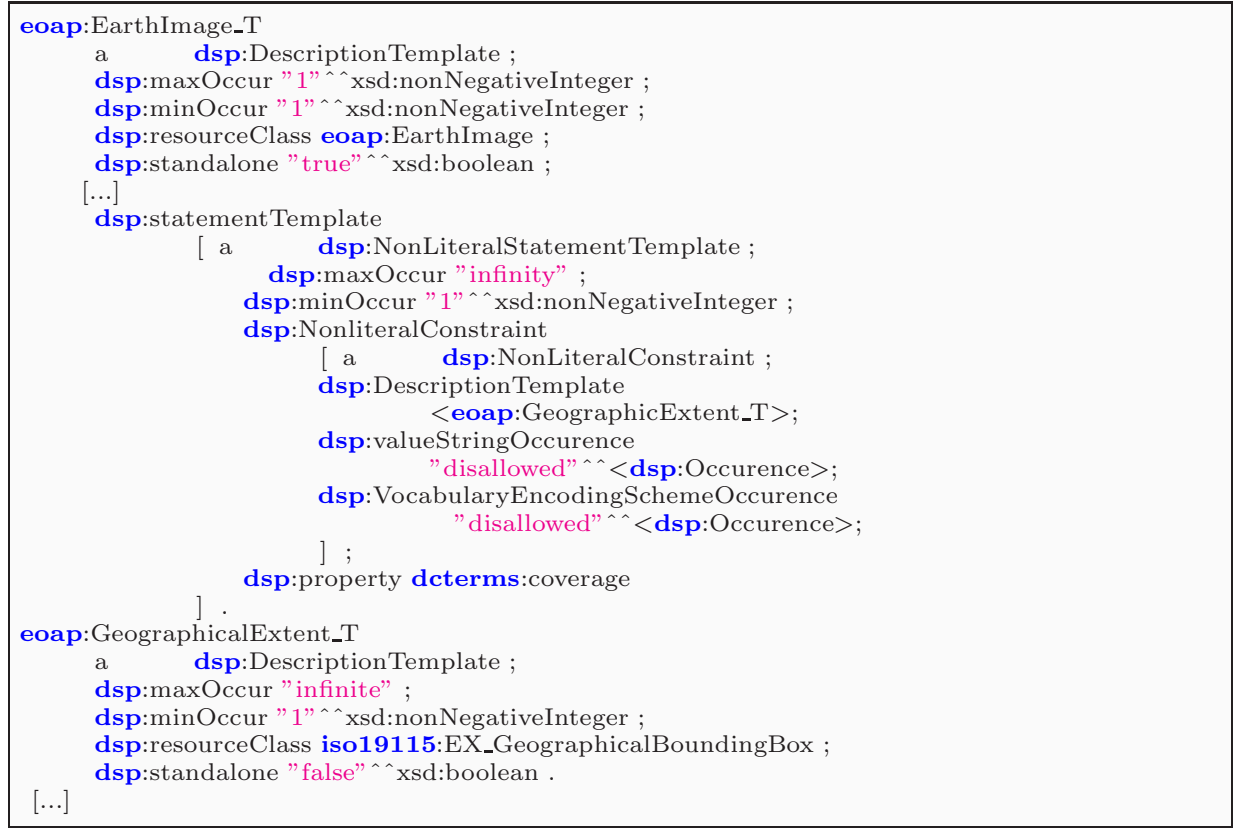

Listing 1.1. code 1 : Statement template coverage. Description Template EarthImage_T sample 
Furthermore, the appropriate description of the entity EarthImage relies on properties only available in the ISO 19115 standard. Such is the case, for example, to describe the spatial resolution of an image or the hierarchical level of the resource described.

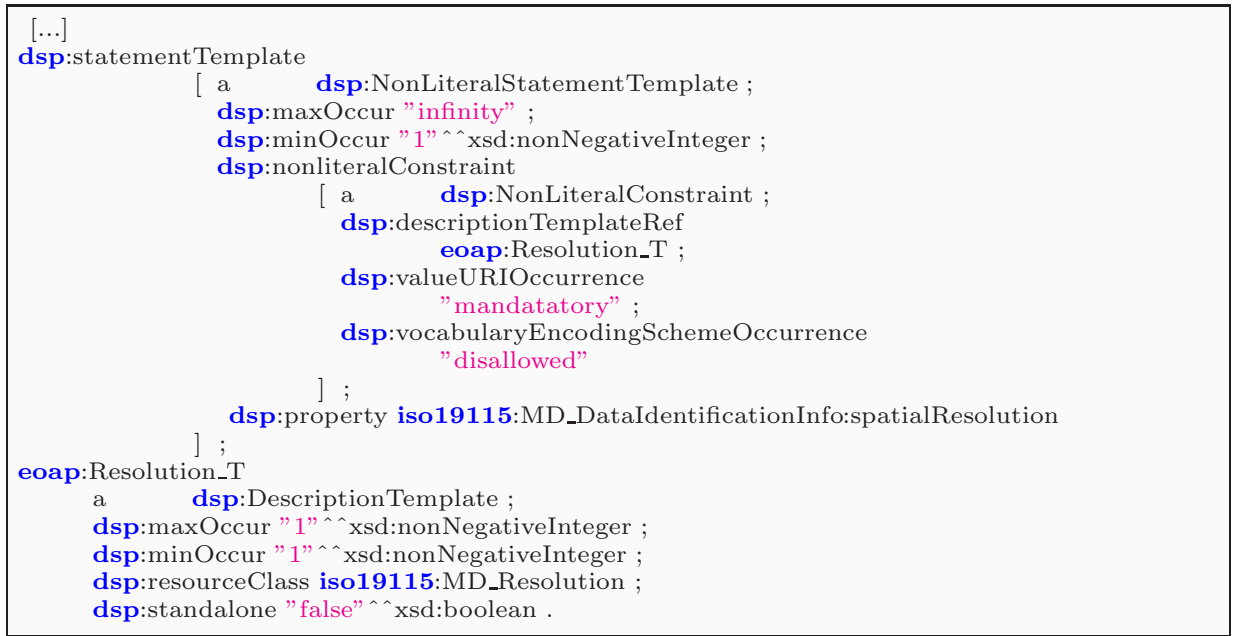

Listing 1.2. code 2 : Statement template spatialResolution. Description Template EarthImage_ $T$ extract

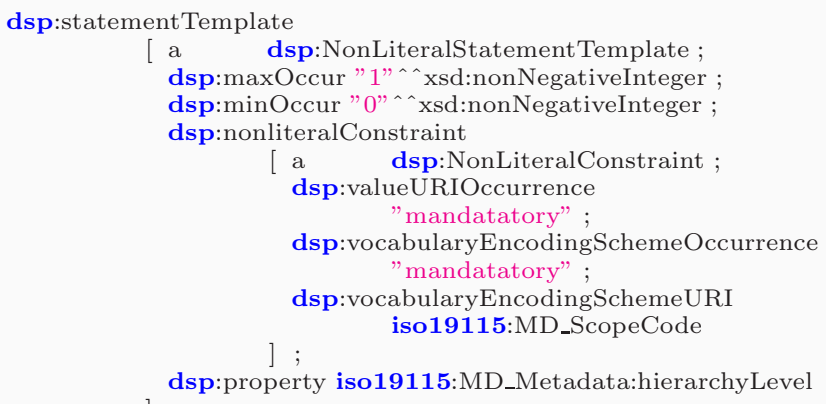

Listing 1.3. code 3 : Statement template hierarchyLevel. Description Template EarthImage_ $T$ extract

We present in Table 5 an extract from the documentation of our Description Set Profile, the Statement Template hierarchyLevel described therein.

User scenario We propose to illustrate the use of the application profile in our system. This scenario that focuses on the discovery of images having properties suitable to meet the demand of a user: a geographer wants to follow the evolution of the urbanization of the city of Montpellier by quantifying the change of the outskirts of the eastern zone, including the passage of land under agri- 


\begin{tabular}{|l|l||}
\hline NAME OF TERM & hierarchyLevel \\
\hline TERM URI & iso19115: MD_Metadata.hierarchyLevel \\
\hline DEFINED BY & http://www.tc211.org/ \\
\hline SOURCE DEFINITION & scope to which the metadata applies \\
\hline EOAP COMMENTS & For satellite image, dataset level and feature [...] \\
\hline VOCAB. ENCODING SCHEME URI & iso19115: MD_ScopeCode \\
\hline OBLIGATION & Optional \\
\hline OCCURRENCE & Not repeatable \\
\hline
\end{tabular}

Fig. 5. Extract of Description Template EarthImage_T - Statement Template hierarchyLevel

cultural influence to a residential area. To identify images that allow him to distinguish different land uses and on which he will conduct his spatial analysis, he needs to ask a question via the discovery service, such as: I'm searching images with a spatial resolution of less than 5 meters, which are located within the bounding rectangle between the coordinates longitude $3,71^{\circ}$; latitude: $43.72^{\circ}$ and coordinates longitude: $4.04^{\circ}$; latitude: $43.53^{\circ}$. The request of the image catalog will be based on properties dcterms : coverage for the spatial extent and iso19115: spatialResolution for spatial resolution written in our DSP. The response provides a set of metadata for images corresponding to the value of these two criteria. We present above, an extract from a DSP instance describing one of the identified images.

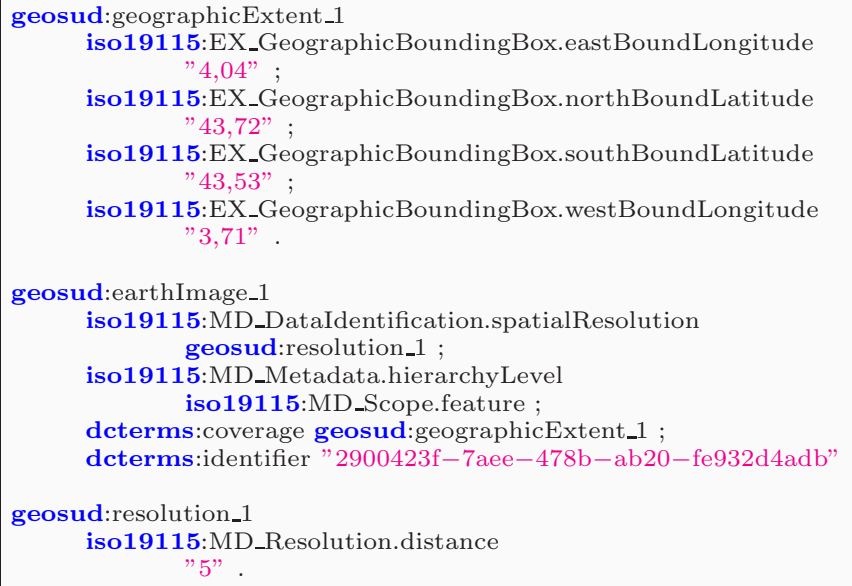

Listing 1.4. Instantiation of Earth Observation DSP

\section{Conclusion and prospects}

This manuscript presents an application profile for Earth observation data. It aims to facilitate sharing of distributed data within a decentralized architecture 
to various user communities. We have retained guidelines from the Singapore Framework to build an open and expandable model reusing existing metadata standards. Therefore the Earth Observation application profile is critical to cover the application requirements such as discovery, location, access, preservation and processing of images underpinning decision activities. The application profile is the core element of the data catalog component within the spatial data infrastructure. In a short-term perspective it is planned to implement the transformation of metadata sets describing the resource from the Earth observation in collections of metadata records compliant to DSP. Likewise, web components are under development using Java EE technologies and will facilitate the building and the management of DSP models. Additionally, they will provide some capabilities to edit, transform and visualize pre-existing or even new metadata records that will enrich newly acquired resources.

Prospects to longer term include:

- Definition of model-based constraints to develop control mechanisms (especially validity) based on the elements of metadata records,

- Harmonization of metadata standards to take advantage of all the metadata sets regardless of their pre-existing format,

- Use of the application profile as an ontology in order to run reasoning on metadata records.

\section{References}

1. Tim Berners-Lee, Dan Connolly, Lalana KagalL, Yosi Scharf, and Jim Hendler. N3Logic: A logical framework for the World Wide Web. Theory and Practice of Logic Programming, 8:249-269, 52008.

2. Carlos Granell and Michael Gould and Miguel Angel Manso and Miguel Angel Bernabé. Handbook of Research on Geoinformatics, chapter V: Spatial Data Infrastructures, pages 36-41. Idea Group Publishing., 2009.

3. Roberto Cossu, Fabrizio Pacini, Fabrice Brito, Luigi Fusco, Eliana Li Santi, and Andrea Parrini. GENESI-DEC: a federative e-infrastructure for Earth Science data discovery, access, and on-demand processing. In 24th International Conference on Informatics for Environmental Protection, 2010.

4. GEO Architecture and Data Committee (version 1.0). GEOSS Core Architecture Implementation Report, 2007.

5. Bernhard Haslhofer and Wolfgang Klas. A survey of techniques for achieving metadata interoperability. ACM Computing Surveys (CSUR), 42(2):7, 2010.

6. Rachel Heery and Manjula Patel. Application profiles: mixing and matching metadata schemas. Ariadne, 25, 2000.

7. Diane I. Hillman, Jon Phipps, and Karen Coyle. Introduction to Application Profiles, 2010

8. Diane I. Hillmann and Jon Phipps. Application Profiles: Exposing and Enforcing Metadata Quality. In Proceedings of the 2007 International Conference on Dublin Core and Metadata Applications: Application Profiles: Theory and Practice, DCMI '07, pages 53-62. Dublin Core Metadata Initiative, 2007.

9. International Organization for Standardization. ISO 19139:2007.

10. International Organization for Standardization. ISO 19156:2011. 
11. International Organization for Standardization. Geographic Information - Metadata. ISO 19115: 2003, May 2003.

12. International Organization for Standardization. Geographic Information - Metadata. ISO 19106:2004, 2004.

13. International Organization for Standardization. ISO 19115-2 - Geographic Information ? Metadata ? Part 2: Extensions for imagery and gridded data. ISO $19115: 2009,2009$.

14. Jérôme Gasperi and Frédéric Houbie and Andrew Woolf. Earth Observation Metadata profile of Observations \& Measurements. OGC Document Number: 10-157r3, 2012.

15. Jo Walsh and Pedro Goncalves. DCLite4G Vocabulary, 2008.

16. Mathieu Kazmierski, Jean-Chistophe Desconnets, Bertrand Guerrero, and Dominique Briand. GEOSUD SDI : Accessing Earth Observation data collections with semantic-based services. In Proceedings of the 17th AGILE Conference on Geographic Information Science, Connecting a Digital Europe through Location and Place, Castellon, Spain, June 2014.

17. Mikael Nilsson. Description Set Profiles: A constraint language for Dublin Core Application Profiles. DCMI Working Draft, 2008.

18. Mikael Nilsson, AlistairJ. Miles, Pete Johnston, and Fredrik Enoksson. Formalizing Dublin Core Application Profiles - Description Set Profiles and Graph Constraints. In Miguel-Angel Sicilia and Miltiadis D. Lytras, editors, Metadata and Semantics, pages 101-111. Springer US, 2009.

19. OGC 07-045. OpenGIS Catalogue Services Specification 2.0.2 - ISO Metadata Application Profile, 2007.

20. OGC 10-032r7. OGC® OpenSearch Geo and Time Extensions, 2013.

21. OSGEO. Geodata metadata model, 2007.

22. OSGEO. Dublin Core Lightweight Profile for Geospatial, 2008.

23. Andy Powell, Mikael Nilsson, Ambjörn Naeve, Pete Johnston, and Thomas Baker. DCMI Abstract Model. DCMI Recommendation, June 2007.

24. Sarah Pulis and Liddy Nevile. Using the DC Abstract Model to support application profile developers. International Conference on Dublin Core and Metadata Applications, 2006. 